\title{
Effective Thermal Conductivity of Nanofluids: Measurement and Prediction
}

\author{
Francisco E. Berger Bioucas ${ }^{1}$ - Michael H. Rausch ${ }^{1}$. Jochen Schmidt ${ }^{2}$. \\ Andreas Bück ${ }^{2}$. Thomas M. Koller ${ }^{1}$ D . Andreas P. Fröba ${ }^{1}$
}

Received: 16 January 2020 / Accepted: 7 February 2020 / Published online: 6 March 2020

(c) The Author(s) 2020

\begin{abstract}
In the present study, the effective thermal conductivity of nanoparticle dispersions, so-called nanofluids, is investigated experimentally and theoretically. For probing the influence of the nanoparticles on the effective thermal conductivity of dispersions with water as liquid continuous phase, nearly spherical and monodisperse titanium dioxide $\left(\mathrm{TiO}_{2}\right)$, silicon dioxide $\left(\mathrm{SiO}_{2}\right)$, and polystyrene (PS) nanoparticles with strongly varying thermal conductivities were used as model systems. For the measurement of the effective thermal conductivity of the nanofluids with particle volume fractions up to 0.31 , a steady-state guarded parallel-plate instrument was applied successfully at temperatures between (298 and 323) K. For the same systems, dynamic light scattering (DLS) was used to analyze the collective translational diffusion, which provided information on the dispersion stability and the distribution of the particle size as essential factors for the effective thermal conductivity. The measurement results for the effective thermal conductivity show no temperature dependency and only a moderate change as a function of particle volume fraction, which is positive or negative for particles with larger or smaller thermal conductivities than the base fluid. Based on these findings, our theoretical model for the effective thermal conductivity originally developed for nanofluids containing fully dispersed particles of large thermal conductivities was revisited and also applied for a reliable prediction in the case of particles of relatively low thermal conductivities.
\end{abstract}

Keywords Dynamic light scattering · Effective thermal conductivity · Measurement · Nanofluids · Parallel-plate method · Prediction

Thomas M. Koller

thomas.m.koller@fau.de

Extended author information available on the last page of the article 


\section{Introduction}

Dispersions of particles on the nanometer scale with a liquid continuous phase, also often called nanofluids, are of interest for many applications of process and energy technology. Here, the miniaturization of electronic devices by efficient cooling [1], the development of high-performance materials for thermal insulation [2], or the design of new working fluids for energy storage [3] can be mentioned. A key property characterizing dispersions is their effective thermal conductivity. First experimental observations in the 1990s claimed that adding a small amount of solid nanoparticles to liquids can enhance the effective thermal conductivity of nanofluids by up to $250 \%$ relative to the base fluid [4]. This report has triggered a plethora of experimental and theoretical studies, most of which give similar conclusions, as it is highlighted in the review article of Tertsinidou et al. [5]. However, other studies do not observe any significant enhancement [5-7]. Until now, debate has continued with respect to the relevant mechanisms affecting thermal conduction in nanofluids, where the Brownian motion [8-10], clustering [11, 12], and interfacial layering $[13,14]$ related to the particles or a combination of these effects were considered as main factors for an enhanced energy transport.

In connection with models for the effective thermal conductivity, theoretical and empirical approaches are available. In the latter, experimental data for the effective thermal conductivity are correlated as a function of relevant parameters characterizing the system such as volume fraction, temperature, particle geometry, and/or layer thickness [15-17]. Among the theoretical models, the most popular model has been developed by Hamilton and Crosser (HC) [18]. Their effective medium theory has originally been derived for two-phase two-component solid dispersions and describes the macroscopic effective thermal conductivity considering the thermal conductivities of the dispersed and continuous phases as well as their relative volume fractions. Based on the $\mathrm{HC}$ model, which is also applicable for nanofluids containing particles of varying size and shape and assumes no thermal resistance at the particle-fluid interface, many different modified versions have been suggested; see, e.g., Refs. [9, 10, 19, 20]. These models include additional effects which in most of the cases aim to explain the significant enhancement of the effective thermal conductivity of nanofluids relative to the thermal conductivity of the base fluid. Our theoretical model [21] for the effective thermal conductivity of nanofluids containing fully dispersed particles accounts for the heat transfer mechanisms caused by thermal conduction in the base fluid and in the particles as well as microconvection due to the Brownian motion of the particles. The consideration of the latter effect at the interface between fluid and particles was found to limit the enhancement of the effective thermal conductivity in comparison with a system where no convective thermal resistance is present. For nanofluids with highly conductive particles of varying size and shape, the predictions from our model were in agreement with the HC model [21]. Thus, our model suggests that the Brownian motion of the particles has no distinct enhancing effect on the effective thermal conductivity of nanofluids. 
The ongoing controversy regarding the actual behavior of the effective thermal conductivity of nanofluids is also connected to the question about the reliability of the experimental methods [5]. The accurate measurement of the thermal conductivity of fluid systems is a challenging task. In addition to the effective thermal conduction through the fluid, which is of primary interest, also other mechanisms of heat transfer in form of, e.g., convection or radiation as well as parasitic heat flows can be present. If not considered in the data evaluation, these effects can falsify the measurements. For dispersions, the measurement results depend not only on the thermodynamic state variables, but also on the measurement procedure itself [5]. According to Tertsinidou et al. [5], the experimental data for the effective thermal conductivity of nanofluids agree well with the predictions from the HC model if data from reliable experimental techniques have been selected.

Existing methods for the measurement of the thermal conductivity can be separated into steady-state and transient methods. Among the latter which are characterized by a local temperature gradient changing as a function of time, the transient hot-wire (THW) technique is most commonly used for nanofluids; see, e.g., Refs. $[5,6,22,23]$. Based on a proper application of the technique, the method allows for an absolute and fast measurement of the thermal conductivity with an expanded uncertainty of about $2 \%$ [6]. Other transient methods including the hot-disk instrument $[24,25]$, the temperature-oscillation method $[15,26]$, or the $3 \omega$-method $[27$, 28] have a limited theoretical basis and often require calibration [5]. Furthermore, the presence of advection during the measurement may affect the obtained effective thermal conductivities. Only a very limited number of experimental investigations on nanofluids is given applying steady-state techniques such as the methods based on concentric cylinders [29-31] or parallel plates [32,33], where the fluid is subjected to a constant temperature gradient during the measurement. Here, for an accurate determination of the thermal conductivity, the Fourier law of heat conduction has to be fulfilled as close as possible in the experimental realization. This includes, for example, a proper guard system around the balanced heating body, which can often not be fulfilled in the above studies. Another imbalance applies to the systems studied experimentally, where the focus lies on nanofluids with particles of relatively large thermal conductivities such as metal oxide particles or carbon nanotubes; see, e.g., Refs. [5, 6, 16, 22, 27]. Only a few experimental studies on the effective thermal conductivity of nanofluids where the particles show similar or smaller thermal conductivities than the base fluid are available [31, 33-37].

The main aim of the present work is to contribute to an improved understanding of the effective thermal conductivity of nanofluids. For this, the effective thermal conductivity of water-based nanofluids with titanium dioxide, silicon dioxide, or polystyrene nanoparticles of strongly varying thermal conductivities was measured with a steady-state guarded parallel-plate instrument. For the characterization of particle size and its distribution as well as the dispersion stability, microscopy and sedimentation measurements were accompanied by dynamic light scattering experiments on the same samples as investigated regarding their effective thermal conductivities. The effects of temperature as well as volume fraction and thermal conductivity of the particles on the measured effective thermal conductivities are discussed and used for the application of our prediction model for the effective 
thermal conductivity of nanofluids, which is also revisited in the present work. This means that the representation of the thermal resistance related to the thermal conduction through the particles was modified appropriately in the model, resulting in an improved prediction of the effective thermal conductivity of nanofluids containing fully dispersed particles.

\section{Experimental}

\subsection{Materials and Sample Preparation}

\subsubsection{Initial Nanofluids}

In the present study, three different types of nanofluids were studied for which the relevant details are given in Table 1 . Two nanofluids are ready-made ones provided by commercial suppliers, while the third one was prepared in our laboratory. The commercial sample “AERODISP ${ }^{\circledR} \mathrm{W}-740 \mathrm{X}$ " from Evonik Industries AG is an aqueous dispersion containing titanium dioxide $\left(\mathrm{TiO}_{2}\right)$ particles with a specified original solid mass fraction $w=0.398$ [38]. In the sample "LUDOX ${ }^{\circledR}$ TM-50 colloidal silica" provided by Sigma Aldrich, silicon dioxide $\left(\mathrm{SiO}_{2}\right)$ particles are dispersed in water with a solid mass fraction $w=0.500[39,40]$. To achieve colloidal stability of the $\mathrm{TiO}_{2}$ and $\mathrm{SiO}_{2}$ nanoparticles with mean diameters of (70 and 22) nm, electrostatic stabilization is adjusted via the $\mathrm{pH}$ which is specified to be 5.9 and 9.0 for the nanofluids containing $\mathrm{TiO}_{2}$ and $\mathrm{SiO}_{2}$, respectively.

For the self-made water-based nanofluid containing polystyrene (PS) particles, the latter were synthesized as described by Zhang et al. [41]. The main steps of the synthesis are briefly summarized below. The PS-based nanofluid was produced by emulsion polymerization. As reaction vessel, a three-neck flask of $250 \mathrm{~mL}$ total volume equipped with a reflux condenser was used. The reaction temperature was controlled by an oil bath heated by a magnetic stirring heating plate. Agitation of the reaction system was performed by a magnetic stirring bar. $0.11 \mathrm{~g}$ of sodium dodecyl sulfate (SDS) as anionic emulsifier and $0.1 \mathrm{~g}$ of potassium persulfate as polymerization initiator were dissolved in $70 \mathrm{~mL}$ of an ethanol-water mixture with an alcoholto-water volume ratio of $5: 2$. Then, $4.5 \mathrm{~mL}$ of styrene was added to the solution. The system was heated to about $343 \mathrm{~K}$ under stirring and kept at this temperature

Table 1 Properties of the initial water-based nanofluids

\begin{tabular}{lllll}
\hline Particle type & $\begin{array}{l}\text { Particle mass } \\
\text { fraction } w\end{array}$ & $\begin{array}{l}\text { Mean particle } \\
\text { diameter/nm }\end{array}$ & Stabilization mechanism & Provider \\
\hline $\mathrm{TiO}_{2}$ & 0.398 & $70^{\mathrm{a}}$ & $\mathrm{pH}(5.9)$ & Evonik Industries AG [38] \\
$\mathrm{SiO}_{2}$ & 0.500 & $22^{\mathrm{a}}$ & $\mathrm{pH}(9.0)$ & Sigma Aldrich [39, 40] \\
$\mathrm{PS}$ & 0.160 & $610^{\mathrm{b}}$ & Surfactant SDS & Self-made \\
\hline
\end{tabular}

\footnotetext{
${ }^{\mathrm{a}}$ Average diameter specified by the supplier

${ }^{\mathrm{b}}$ Average number-based diameter obtained from sedimentation analysis
} 
for $8 \mathrm{~h}$ to allow for complete polymerization. The mass fraction of PS after synthesis was about 0.06. Finally, the product was washed several times with deionized water by centrifugation and concentrated to a mass fraction of PS particles of $w=(0.165 \pm 0.002)$ for further usage. Here, the concentration of the surfactant SDS in the nanofluid can be estimated to be smaller than $10^{-4} \mathrm{M}$. Although the mean diameter of the dispersed PS particles of $610 \mathrm{~nm}$ is larger than $100 \mathrm{~nm}$ and, thus, does not strictly apply to the definition of a nanofluid, we keep the term "nanofluid" also in connection with the PS-based nanofluids throughout the entire manuscript for the sake of convenience.

For the three types of initial nanofluids, the relative expanded $(k=2)$ uncertainty of the mass fraction of the dispersed particles $(k=2)$ is estimated to be $5 \%$. Furthermore, the volume fraction of the stabilizing agents and other impurities originating from the manufacturing process is considered to be smaller than $10^{-3}$ and, thus, sufficiently small to have a negligible impact on the properties including the effective thermal conductivity of the studied nanofluids.

\subsubsection{Preparation of Diluted Nanofluids}

Dilution of the initial nanoparticle dispersions was realized with distilled water to prepare nanofluids with different particle volume fractions $\varphi$. For this, a flask was filled with distilled water to which the required amount of the initial nanofluid was added using a syringe. Before this, each nanofluid sample was shaken manually and then sonicated for about $15 \mathrm{~min}$ in an ultrasonic bath to obtain a homogeneous suspension. The concentrations of the diluted nanofluids were determined by weighing the flasks before and after adding the nanoparticle dispersion with a Sartorius Entris 224I-1S balance with a precision of $0.1 \mathrm{mg}$ and an estimated expanded uncertainty $(k=2)$ of $1 \mathrm{mg}$. For each nanofluid type, the mass fraction of particles in the diluted dispersions was adjusted to be about a half and a fourth of that in the initial dispersion. These three dispersions referred to as "concentrated nanofluids" in the following with particle volume fractions between about $4 \cdot 10^{-2}$ and $3 \cdot 10^{-1}$ have been used in connection with the thermal conductivity and dynamic light scattering (DLS) measurements. Furthermore, two "highly diluted nanofluids" with very low particle volume fractions on the order of $1 \cdot 10^{-6}$ and $2 \cdot 10^{-5}$, i.e., close to infinite dilution, were prepared to obtain information on the particle size by DLS. The mass fractions of particles were converted to volume fractions based on the density of the pure bulk fluid water $\left(\rho_{\mathrm{bf}}\right)$ [42] and of the particles $\left(\rho_{\mathrm{p}}\right)$. In this approach, an ideal mixing behavior neglecting any influences of further impurities is assumed. Since the true density of the particles is not specified by the suppliers or is unknown, literature data at $298.15 \mathrm{~K}$ were collected for $\mathrm{TiO}_{2}\left(\rho_{\mathrm{p}}=3900 \mathrm{~kg} \cdot \mathrm{m}^{-3}\right)$ [6], $\mathrm{SiO}_{2}\left(\rho_{\mathrm{p}}=2196 \mathrm{~kg} \cdot \mathrm{m}^{-3}\right)$ [43], and PS $\left(\rho_{\mathrm{p}}=1050 \mathrm{~kg} \cdot \mathrm{m}^{-3}\right)$ [44]. For the temperature range investigated here, the temperature dependence of the true density of the particles can be neglected. Taking into consideration the sample preparation procedure and the uncertainties associated with the input parameters, the relative expanded $(k=2)$ uncertainty of the particle volume fractions in all nanofluids studied is estimated to be $5 \%$. 
All nanofluid samples used in connection with the characterization and measurement methods described in the following Sects. 2.2 and 2.3 were stored within flasks inside a refrigerator at a temperature of about $277 \mathrm{~K}$. Prior to these investigations, the samples were dispersed in an ultrasonic bath for about $15 \mathrm{~min}$ to improve dispersion.

\subsection{Characterization of Nanofluids}

\subsubsection{Visual Inspection of Stability}

For probing the long-time stability of the nanofluids investigated in connection with the effective thermal conductivity, $10 \mathrm{~mL}$ of a freshly prepared and sonicated sample inside a $10 \mathrm{~mL}$ vial was kept at ambient conditions at a temperature of about $293 \mathrm{~K}$ over a period of 13 days. During this time, a daily visual inspection was performed for all concentrated nanofluids. For all $\mathrm{TiO}_{2}$ - and $\mathrm{SiO}_{2}$-based samples, no visual sedimentation was observed. This has also been found by Ehle et al. [31] for the same type of initial $\mathrm{SiO}_{2}$-based nanofluid over a period of 17 days. For the nanofluids containing PS particles where corresponding photographs at days 1, 2, 3, 4, and 13 are shown in Fig. 1, a weak sedimentation effect was observed after about 4 days for the initial suspension and after 3 days for the two diluted suspensions. The earlier sedimentation of the two diluted nanofluids can be related to the varying concentration of the SDS surfactant which is reduced in the same way as the nanoparticle concentration upon dilution by water. Nevertheless, for the time period of the measurements of the effective thermal conductivity lasting around 2 days per sample, stability can be assumed for all nanofluids studied.

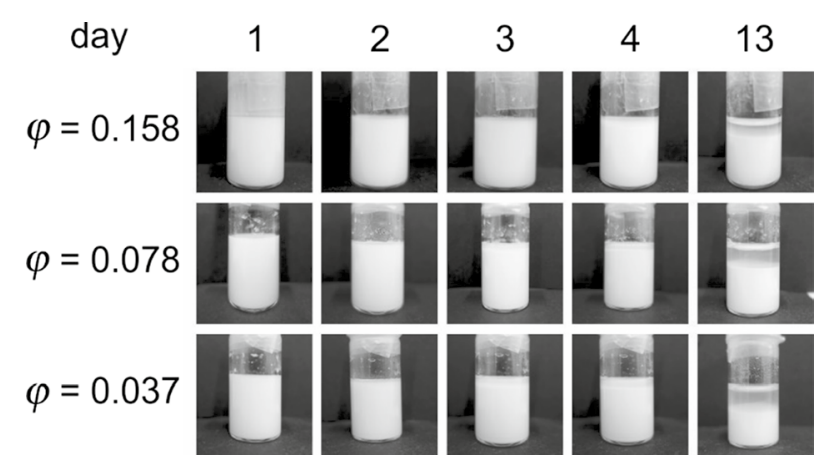

Fig. 1 Photographs of the concentrated water-based nanofluids containing PS particles for various days after the preparation and sonication of the initial $(\varphi=0.158)$ sample and the diluted $(\varphi=0.078$ and 0.037) samples 

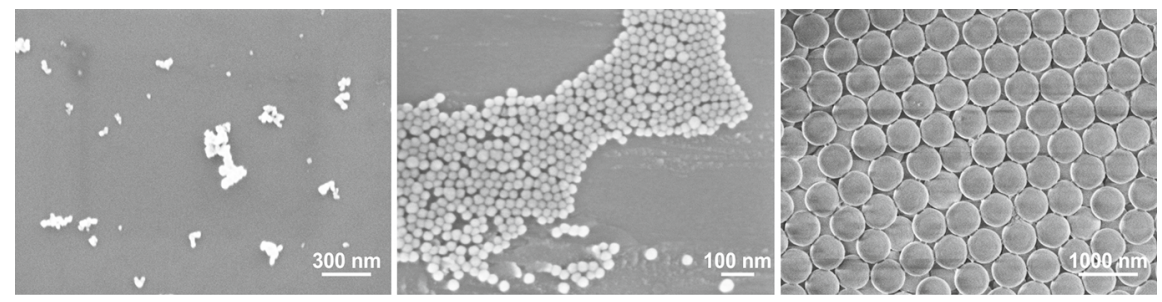

Fig. 2 SEM micrographs of the dried $\mathrm{TiO}_{2}$ (left), $\mathrm{SiO}_{2}$ (middle), and $\mathrm{PS}$ (right) nanoparticles

\subsubsection{Scanning Electron Microscopy}

The size and shape of the particles was assessed by scanning electron microscopy (SEM). For sample preparation, the initial nanofluids were diluted to particle volume fractions of about 0.005 . From the diluted samples, about $10 \mu \mathrm{L}$ suspension was deposited onto an air plasma-treated silicon wafer and dried at ambient temperature over night. The SEM-device Gemini Ultra 55 from Zeiss is equipped with a through-the-hole detector and was operated at an acceleration voltage of $1 \mathrm{kV}$. For the three particle types studied, exemplary micrograph images are shown in Fig. 2 and used for particle size analysis after their binarization where only isolated particles were considered. The $\mathrm{SiO}_{2}$ and PS particles are of almost perfect spherical shape and narrow size distribution with average primary particle diameters including double standard deviations $(k=2)$ of $(24.2 \pm 4.2) \mathrm{nm}$ and $(565 \pm 54) \mathrm{nm}$, respectively. In contrast, the $\mathrm{TiO}_{2}$-based system is made up of aggregates of differently sized primary particles with a diameter of about $70 \mathrm{~nm}$ which is also specified by the supplier as average particle diameter [38]. The aggregates were supposed to be formed during the drying process for the preparation of the SEM analysis.

\subsubsection{Differential Centrifugal Sedimentation}

To obtain further information about the morphology of the initial concentrated nanofluids and for comparison with the results from SEM, the particle size distributions of the considered materials were determined via differential centrifugal sedimentation analysis. For this, a DC2400 disk centrifuge from CPS Instruments operated at $24000 \mathrm{~min}^{-1}$ was applied. For the nanofluids containing $\mathrm{SiO}_{2}$ and $\mathrm{TiO}_{2}$, an aqueous sucrose density gradient with nine sucrose mass fractions between 0.08 and 0.24 in steps of 0.02 was used, while smaller sucrose density gradients at nine mass fractions ranging from 0 to 0.08 in steps of 0.01 were applied for the PS-based nanofluid. The density gradients were realized by successive injection of $1.6 \mathrm{~mL}$ of nine sucrose solutions starting with the largest concentration. Prior to the differential sedimentation analysis, the density gradient was equilibrated for $15 \mathrm{~min}$. As particle size standard, spherical polyvinylchloride (PVC) particles with a diameter of $483 \mathrm{~nm}$ purchased from CPS Instruments were used.

The volume sum distributions $Q_{3}$ of the considered materials determined by sedimentation analysis are depicted in Fig. 3. In general, the results from SEM imaging and/or from the suppliers are confirmed. For PS, a volume-averaged particle 


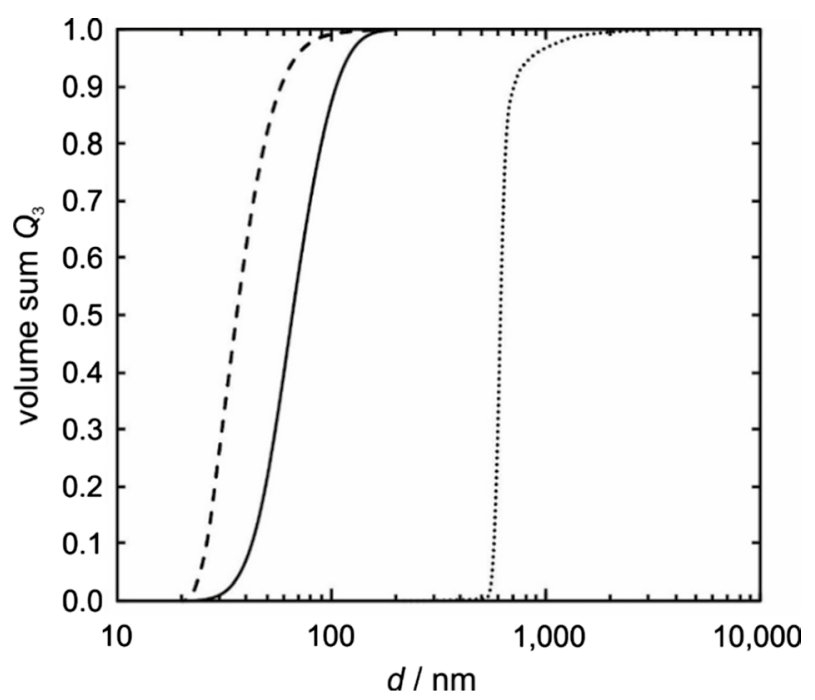

Fig. 3 Volume sum distributions for the investigated particles in the initial nanofluids obtained by differential centrifugal sedimentation analysis: , $\mathrm{TiO}_{2} ;-$ -, $\mathrm{SiO}_{2} ; \ldots . ., \mathrm{PS}$

diameter $d_{50,3}=610 \mathrm{~nm}$ including the lower bound $d_{10,3}=569 \mathrm{~nm}$ and the upper bound $d_{90,3}=705 \mathrm{~nm}$ was obtained. The very narrow size distribution of the PS particles is expressed by the span $\left(d_{90,3}-d_{10,3}\right) \cdot\left(d_{50,3}\right)^{-1}$ of 0.22 . For $\mathrm{TiO}_{2}$ and $\mathrm{SiO}_{2}$, diameters of $d_{50,3}=66 \mathrm{~nm}\left(d_{10,3}=43 \mathrm{~nm}, d_{90,3}=105 \mathrm{~nm}\right)$ and $d_{50,3}=36 \mathrm{~nm}$ $\left(d_{10,3}=26 \mathrm{~nm}, d_{90,3}=58 \mathrm{~nm}\right)$ were obtained. Compared to the PS particles, the widths of the particle size distributions for the two oxidic particle types is larger with spans of 0.94 for $\mathrm{TiO}_{2}$ and 0.88 for $\mathrm{SiO}_{2}$ particles.

\subsection{Measurement Methods}

\subsubsection{Effective Thermal Conductivity by Guarded Parallel-Plate Instrument}

The effective thermal conductivity of the nanofluids $\lambda_{\text {eff }}$ was measured at ambient pressure with a guarded parallel-plate instrument. A detailed description of the complete measurement instrument including its application for the investigation of fluids and solids can be found in Refs. [45-47]. Below, only essential features of the instrument and the experimental conditions and procedures relevant for this study are given.

The principle of parallel-plate instruments for the measurement of the thermal conductivity $\lambda$ is to fulfill the ideal one-dimensional form of the Fourier law of heat conduction for a planar sample,

$$
\dot{Q}=A \frac{\lambda}{S}\left(T_{\text {hot }}-T_{\text {cold }}\right),
$$

in the experimental realization as close as possible. For this, it is necessary to perform a precise determination of the heat flux $\dot{Q}$ caused purely by conduction through 
the sample, the heat transfer area $A$, the sample layer thickness $s$, and the temperature difference $\Delta T\left(=T_{\text {hot }}-T_{\text {cold }}\right)$ between the two outer surfaces of the sample. For an accurate measurement of the thermal conductivity, any parasitic heat fluxes due to convection in the sample, heat leakages to the surrounding, radiative heat transfer between the plates contacting the sample, and further effects related to the Soret and/or Dufour effect of the particles have to be minimized or considered reliably, which is discussed below.

The sample is placed between two parallel circular plates oriented perpendicular to the gravitational field. By this arrangement and the heat flux from the upper to the lower plate, advection effects inside the sample layer are minimized. For all experimental conditions in the present study, calculated Rayleigh numbers were in a range between 200 and 530 for pure water and between 3 and 400 for the nanofluids, which is smaller than the critical value of 1000 stated by Naziev et al. [48] for vertical coaxial cylinders. Thus, advective heat transfer can be neglected for all measurements. To avoid any heat leakages from the balancing heating plate (diameter $85.96 \mathrm{~mm} \pm 0.01 \mathrm{~mm}$ ) to the surrounding, the plate is immersed in a multi-part guard system. Each component of the guard system is controlled independently to the same temperature as the heating plate. The temperature control is realized by resistance heating for the heating plate and the guard components as well as by Peltier elements for the cooling plate. For the Pt100 resistance probes used for all temperature measurements in the instrument, a calibration of the probes including the whole cabling of the four-wire system was performed, resulting in an absolute uncertainty $(k=2)$ of $0.01 \mathrm{~K}$. As a further guard around the multi-guard system, an insulating housing was kept at about $2 \mathrm{~K}$ below the temperature of the heating plate by a lab thermostat.

A possible contribution of radiative heat transfer between the plates to the measured overall heat flux through the sample is minimized by covering the polished surface of the plates with a thin chrome layer to reduce the emission coefficient to about 0.04. For pure water as a relatively strongly absorbing fluid, radiation contributions can be neglected because they make up less than $0.1 \%$ of the measured thermal conductivities [47]. Since the investigated water-based nanofluids become more and more opaque with increasing particle concentration, absorption of radiation is expected to be more pronounced than for pure water. Thus, any influence of radiative heat transfer on the measurement results can be neglected for all systems studied in this work.

For nanofluid systems, besides a conductive energy transport caused by a temperature gradient, also energy transport caused by a concentration gradient, the socalled Dufour effect, may arise. The latter effect results from the mass transfer of the particles due to the applied temperature gradient. Ali Pakravan and Yaghoubi [49] derived an analytical expression for the energy transport due to the Dufour effect, which increases with increasing particle thermodiffusion coefficient and temperature and with decreasing self-diffusion coefficient and volume fraction of the particles. For typical values of the thermodiffusion and self-diffusion coefficient of nanoparticles on the order of $10^{-13} \mathrm{~m}^{2} \cdot \mathrm{s}^{-1} \cdot \mathrm{K}^{-1}$ and $10^{-11} \mathrm{~m}^{2} \cdot \mathrm{s}^{-1}$ [50], the contribution to the effective thermal conductivity of the present nanofluids caused by the Dufour effect is distinctly less than $1 \%$ and, thus, can be neglected. According to the numerical calculations of Savino et al. [51], steady-state techniques such as the parallel-plate 
method are also not affected by thermodiffusion effects because in principle the entire fluid volume is subjected to the balancing heat flux. Although a concentration gradient could be present over the sample layer if the particle thermodiffusion coefficient is unequal zero, the measurement result is related to an average effective thermal conductivity corresponding to the overall composition of the nanofluid. This can, however, only be ensured if $\lambda_{\text {eff }}$ shows a linear dependence on the particle volume fraction, which holds well for nanofluids [5].

Within the sample preparation and measurement procedure, first, the thickness of the sample layer was adjusted to $(1 \pm 0.02) \mathrm{mm}(k=2)$ for all reported measurements. The filling of the sample layer was performed with a peristaltic pump by circulating the water or nanofluid sample until no air bubbles were observed from the outlet channel. Then, the sample gap was closed with bellow-type valves. For the measurement, the sample was subjected to a constant temperature gradient related to a defined temperature difference between the upper heating plate and the lower cooling plate. Once the temperatures of these two plates including the multi-guard system were stable within $0.01 \mathrm{~K}$, the resulting heat flux conducted through the sample is determined by measuring the electric power dissipated to the resistance heating wire with a relative uncertainty $(k=2)$ of $0.2 \%$. For the heating plate, the temperatures measured at distances of ( 5 and 13) $\mathrm{mm}$ from its surface are averaged and used as surface temperature, given the large thermal conductivity of the copper material and the low heat flux. In the cooling plate, the data from only one temperature probe installed with a distance of $5 \mathrm{~mm}$ from the surface is directly used as surface temperature. Based on Eq. 1, the effective thermal conductivity of all investigated samples is determined in an absolute way with an estimated expanded uncertainty of $5 \%(k=2)$, taking into consideration the uncertainties of the input parameters.

As a reference, the thermal conductivity $\lambda_{\mathrm{bf}}$ of the bulk fluid water was measured at ambient pressure at mean temperatures of $(298.15,303.15,313.15$, and 323.15) $\mathrm{K}$ using two temperature differences of (2 and 3) K between the two plate surfaces. Thereafter, the effective thermal conductivity $\lambda_{\text {eff }}$ of the nine different nanofluids was measured at the same four mean temperatures as studied for pure water using a temperature difference of $3 \mathrm{~K}$. Here, the measurements for a given sample were performed from the lowest to the highest temperature within 1 day. On the next day, a repetition measurement was performed at $303.15 \mathrm{~K}$ as a cross-check. Then, the instrument was opened and the sample was inspected visually for any indications of sedimentation or agglomeration in the nanofluids, which could not be observed in all cases. After the investigation of each nanofluid sample, the cleaned instrument was tested by measuring the thermal conductivity of water at the four aforementioned temperatures with a temperature difference of $3 \mathrm{~K}$. In all cases, agreement with the first measurement set within the expanded experimental uncertainty of the measured data was found.

\subsubsection{Diffusion Coefficients by Dynamic Light Scattering (DLS)}

For the characterization of particle size and its distribution as well as the dispersion stability of the nanofluids in the same fluid state as it is investigated in connection with the effective thermal conductivity, the diffusion coefficients were obtained by dynamic light scattering (DLS). For details on the principles of the technique 
[52] and its application for the determination of several thermophysical properties of various types of fluids [53-56] and dispersed systems [57-59], the reader is referred to the respective literature. In the following, only the information relevant for the present investigations is given.

For nanofluids in macroscopic thermodynamic equilibrium, the decay of microscopic fluctuations in the particle concentration is governed by the translational particle diffusion coefficient $D$ and reflected in the time-dependent intensity correlation function $(\mathrm{CF})$ of light scattered by the particles. For monodisperse spherical particles and heterodyne conditions where the scattered light is superimposed coherently with reference light of much larger intensity, the detectable normalized $\mathrm{CF}$ takes the form of an exponential with a characteristic decay time $\tau_{\mathrm{C}}$ [60]. The latter represents the mean lifetime of the observed fluctuations and is connected to $D$ by $\tau_{\mathrm{C}}=\left(D \cdot q^{2}\right)^{-1}$. The modulus of the scattering vector $q=\left(4 \pi n / \lambda_{0}\right) \cdot \sin \left(\Theta_{\mathrm{S}} / 2\right)$ is given by the scattering angle $\Theta_{\mathrm{S}}$ between the directions of the incident and the scattered light and by the refractive index $n$ of the fluid at the laser wavelength in vacuo $\lambda_{0}=(532.1 \pm 0.3) \mathrm{nm}$. For polydisperse samples featuring a distribution of particle sizes, the CF represents a spread of exponentials with different decay times and signal intensities. Here, a cumulant analysis for the calculation of the decay time distribution is often employed [60].

For mono- or polydisperse particles dispersed in Newtonian fluids close to infinite dilution at $\varphi$ values smaller than about $10^{-4}$, the measured translational diffusion coefficients can be used to determine a hydrodynamic diameter or its distribution via the Stokes-Einstein equation [61], $D=\left(k_{\mathrm{B}} T\right) /(3 \pi \eta d)$. Here, $k_{\mathrm{B}}$ is the Boltzmann constant, $T$ is the absolute temperature, $\eta$ is the dynamic viscosity of the solvent, and $d$ is the effective hydrodynamic diameter of the particles. For concentrated dispersions far away from infinite dilution, the Stokes-Einstein equation is not valid anymore, but still often used in literature [24, 25, 62] for the determination of particle size. In such dispersions, direct particle interactions and indirect hydrodynamic interactions affect the collective diffusion coefficient $D$ in the particle-fluid system and, thus, the relaxation times observed by DLS $[63,64]$. In related theoretical approaches, $D$ is commonly represented in form of a virial expansion with respect to the particle volume fraction $[63,65]$. A further experimental challenge is that with increasing particle concentration, multiple scattering between the particles can occur, which further impedes the interpretation of DLS data [63].

In the present study, the same experimental setup as applied in our previous work [59] has been used to perform heterodyne DLS experiments under vertical polarization directions of the incident and the detected light. For the concentrated nanofluids which are strongly light-absorbing in most cases, only a detection of the scattered light in reflection direction was possible. Here, the detection volume was positioned close to the wall of the cuvette, which enables the application of low laser powers and minimizes multiple-scattering effects. Under such conditions, five scattering angles between (146 and 163$)^{\circ}$ were adjusted. For the highly diluted nanofluids, transparent samples are given, which allowed to analyze the scattered light under a scattering angle of $\Theta_{\mathrm{S}}=90^{\circ}$. In all cases, an expanded uncertainty $(k=2)$ in $\Theta_{\mathrm{S}}$ of $1^{\circ}$ can be specified. The sample temperature was measured by a calibrated Pt100 resistance probe with an expanded uncertainty $(k=2)$ of $0.04 \mathrm{~K}$. Within one measurement 
series, i.e., five measurements at different scattering angles for the concentrated nanofluids or five independent measurements at $\Theta_{\mathrm{S}}=90^{\circ}$ for the highly diluted nanofluids, the temperature stability was always better than $\pm 0.014 \mathrm{~K}$. A sample of each concentrated nanofluid which was filled into the guarded parallel-plate instrument was investigated at (298.15 and 323.15) K, with a repetition at $298.15 \mathrm{~K}$ within one day. One day after the thermal conductivity measurements were finished, the corresponding sample was removed from the instrument and analyzed by DLS at $298.15 \mathrm{~K}$ to compare the results with those from the previous day.

For the data evaluation detailed in our previous work on highly diluted nanoparticle dispersions [59], the CFs measured simultaneously by the linear-tau and multitau correlator were represented by least-squares fitting. In all cases, the sum of two or three exponentially decaying signals associated with the corresponding mean decay times $\tau_{\mathrm{C}}$ were needed to obtain residuals between the measured and fitted data, which are free of systematic behaviors. For comparison, also an analysis of the distribution of decay times determined via the cumulant analysis for the corresponding correlator data obtained by the multi-tau correlator was performed. In all measurements reported here, agreement between the two analysis methods was found.

For the refractive index $n$ of the nanofluids required to obtain $q$, the volume fraction-based Gladstone-Dale mixing rule $[66,67]$ considering the refractive indices of pure water and the particles was used as an estimation for the concentrated nanofluids. This rule agreed within the estimated uncertainty with the experimental data obtained with an Abbe refractometer detailed in Ref. [68]. In the present study, measurements were only possible for the relatively transparent nanofluids with $\mathrm{SiO}_{2}$ at all volume fractions or with $\mathrm{TiO}_{2}$ up to $\varphi=0.02$. In case of the highly diluted nanofluids, the refractive index of pure water could reliably be used. For $\lambda_{0}=532.1 \mathrm{~nm}, n$ data for water as a function of temperature [69] as well as temperature-independent data for $\mathrm{TiO}_{2}$ [70], $\mathrm{SiO}_{2}$ [70], and PS [71] were employed. The refractive index data are calculated at the average temperatures of the related DLS measurement series with an estimated relative expanded uncertainty of $0.5 \%$ and $2 \%$ for the highly diluted and concentrated nanofluids. For each thermodynamic state, the final diffusion coefficients were obtained by averaging the data determined from the individual CFs recorded with the two correlators for five different individual measurements using an uncertainty-based weighting scheme [59]. As expanded uncertainties of the $D$ data, the double standard deviations of the values obtained from the individual measurements and weighted in the same manner were calculated.

\section{Results and Discussion}

In the following, the measured data for the translational diffusion coefficients obtained by DLS and used to characterize the stability and hydrodynamic diameter of the nearly spherical particles dispersed in water are summarized. Then, the measurement results for the thermal conductivity of pure water and the effective thermal conductivity of the nanofluids including corresponding enhancement factors are discussed and compared with available literature data and common theoretical 
approaches. Finally, an improved analytical model for the effective thermal conductivity of nanofluids is proposed.

\subsection{Diffusion Coefficients}

Figure 4 shows the mean diffusion coefficients $D$ of the water-based nanofluids measured by DLS at a temperature of $298.15 \mathrm{~K}$ as a function of the volume fractions

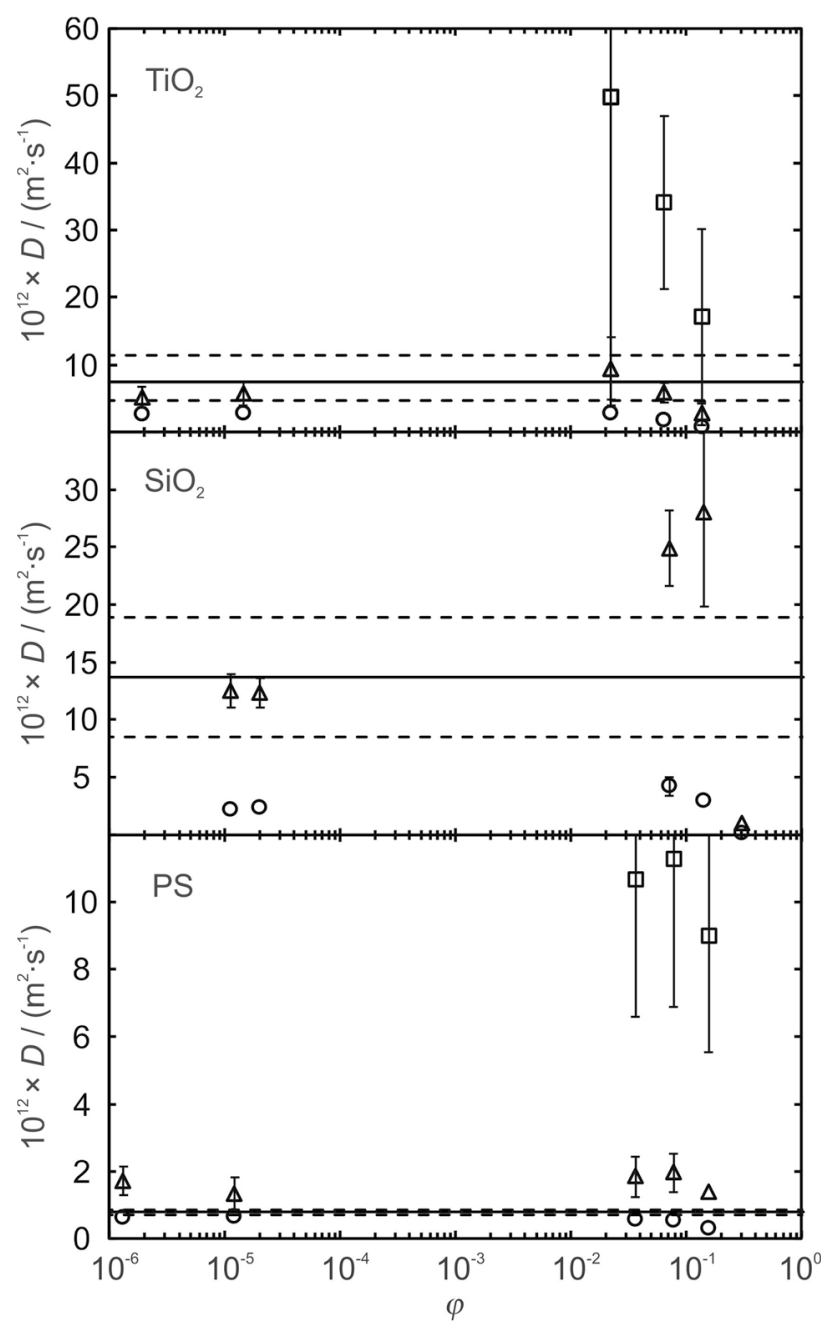

Fig. 4 Diffusion coefficients $D$ in the water-based nanofluids measured by DLS at a temperature of $298.15 \mathrm{~K}$ as a function of the volume fractions of the dispersed $\mathrm{TiO}_{2}$ (upper part), $\mathrm{SiO}_{2}$ (middle part), and PS (lower part) particles. The symbols refer to the mean diffusion coefficients related to the measured mean decay times of the fast $(\square)$, medium $(\Delta)$, and slow $(\bigcirc)$ exponential signals. The lines and dashed lines indicate the values calculated by the Stokes-Einstein equation [61] at conditions close to infinite dilution of the particles considering their average diameters and lower or upper boundaries evaluated by the sedimentation analysis 
of the dispersed $\mathrm{TiO}_{2}$ (upper part), $\mathrm{SiO}_{2}$ (middle part), and $\mathrm{PS}$ (lower part) particles. For each system, besides the two highly diluted samples with particle volume fractions below $10^{-4}$, i.e., close to infinite dilution, also the results for three concentrated nanofluids investigated in connection with the effective thermal conductivity are given.

For all systems at conditions close to infinite dilution discussed in the following, two main modes related to two diffusion coefficients were observed. Each of the diffusion coefficients agrees within combined uncertainties at the two studied particle volume fractions. For comparison, theoretical translational diffusion coefficients shown by the solid lines were calculated based on the Stokes-Einstein equation [61]. For this, we used the reference data for the dynamic viscosity of pure water reported by Huber et al. [72] and the volume-averaged particle diameter $d_{50,3}$ which was obtained from the sedimentation analysis and is approximated as hydrodynamic diameter. The dashed lines indicate the predictions, where the lower $\left(d_{10,3}\right)$ and upper $\left(d_{90,3}\right)$ boundaries of the evaluated particle diameters were used. Within combined uncertainties, agreement between the theoretical $D$ data and one of the two measured diffusion coefficients is found for each system. The mean hydrodynamic diameters of $85.2 \mathrm{~nm}, 39.3 \mathrm{~nm}$, and $877.9 \mathrm{~nm}$ for $\mathrm{TiO}_{2}$ (triangles), $\mathrm{SiO}_{2}$ (triangles), and PS (circles), respectively, obtained from the diffusion coefficients from DLS for the lowest particle volume fractions fit well with the mean particle diameters deduced from the sedimentation analysis, but also with those from SEM at diluted conditions. The somewhat larger diameters obtained from DLS than the ones from SEM may be related to the additional hydrodynamic layer around the particles. As can be seen in Fig. 4, the smaller diffusion coefficients for the systems with $\mathrm{TiO}_{2}$ or $\mathrm{SiO}_{2}$ (circles) seem to be related to the presence of larger particle aggregates resulting from the changing $\mathrm{pH}$ values by water dilution. For the systems with PS, the additional faster diffusion coefficients (triangles) may be artifacts caused by multiple-scattering effects which give rise to an additional fast decaying contribution in the measured CF.

For the concentrated nanofluids at particle volume fractions larger than $10^{-2}$, the two main diffusion coefficients apparently related to the collective diffusion of single and aggregated particles are still observable for all systems. Both diffusion coefficients are almost independent of concentration and tend to increase weakly up to volume fractions of about $10^{-1}$, which is in agreement with the common theories $[63,65,73]$. At the largest particle volume fractions studied for each system, a distinct reduction of the measured diffusion coefficients can be found, in particular for the most concentrated $\mathrm{SiO}_{2}$-based nanofluid with $\varphi=0.312$. The increasing impact of interparticle interactions with increasing particle fraction are often reflected in increasing viscosities and decreasing diffusion coefficients in concentrated dispersions [73]. For the $\mathrm{TiO}_{2}$ - and PS-based systems, an additional fast DLS signal of similar intensity as the other two signals was observed. The respective pseudo diffusion coefficients indicated by the square symbols in Fig. 4 seem to be related to multi-scattering effects which are more dominant for larger concentrations and larger particles, as it is given for $\mathrm{TiO}_{2}$ and especially PS. Furthermore, effects caused by many-body excluded volume, hydrodynamic interactions, polydispersity with respect to size and shape, and/or additional rotational motion besides 
translational motion impede the interpretation of DLS measurements at intermediate and high concentrations [74], which is outside the scope of the present work. Future investigations should analyze the different light scattering signals for concentrated dispersions.

The DLS experiments performed at $323.15 \mathrm{~K}$ revealed the same concentrationdependent trends as shown in Fig. 4 at 298.15 K. For the larger temperature, also comparable hydrodynamic diameters within combined uncertainties could be calculated at conditions close to infinite dilution. For all systems, the results from the repetition measurements at $298.15 \mathrm{~K}$ performed at the same day for the same sample after the measurement at $323.15 \mathrm{~K}$ and at the next day for the sample investigated within the guarded parallel-plate instrument agree with the results given in Fig. 4 within combined uncertainties. All these observations imply a high degree of stability of the probed nanofluids over the entire concentration range investigated.

\subsection{Effective Thermal Conductivity}

\subsubsection{Summary of Measurement Results}

For the base fluid water, the measured thermal conductivities $\lambda_{\text {bf }}$ obtained for temperatures from (298.15 to 323.15$) \mathrm{K}$ at atmospheric pressure are shown in Fig. 5. Two temperature differences between the sample surfaces of (2 and 3) $\mathrm{K}$ were applied for all temperature states except for $323.15 \mathrm{~K}$ where only $\Delta T=3 \mathrm{~K}$ was investigated. All data obtained with the guarded parallel-plate instrument deviate by less than $7 \%$ from the reference data [75], which is within the combined expanded uncertainties of the datasets. The positive deviations are in agreement with our former measurement results [47] and seem to be attributed to underestimated heat leakages between the heating plate and the guard system. These leakages are suppressed as much as possible by the use of a multi-guard system around the heating plate. Their complete elimination represents a challenging task and can hardly be realized

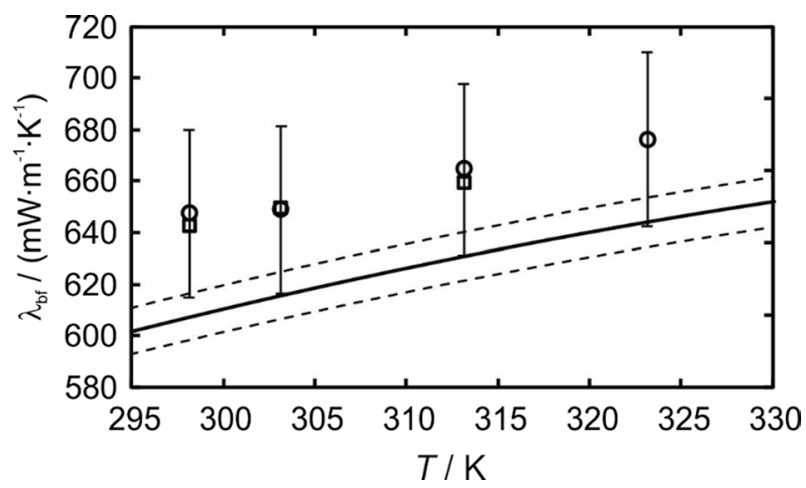

Fig. 5 Thermal conductivity of water at atmospheric pressure as a function of temperature: $\square(\Delta T=2 \mathrm{~K})$, $\mathrm{O}(\Delta T=3 \mathrm{~K})$, this study, measurements; —_, Huber et al. [75], reference correlation. For the experimental data, the error bars representing the expanded measurement uncertainties $(k=2)$ are only shown for $\Delta T=3 \mathrm{~K}$. Dashed lines represent the expanded uncertainties of the reference correlation $(k=2)$ 
Table 2 Measurement results for the thermal conductivity of water and the effective thermal conductivity of the studied water-based nanofluids including the corresponding enhancement factors

\begin{tabular}{|c|c|c|c|c|c|}
\hline System & $w$ & $\varphi$ & $T / \mathrm{K}$ & $\begin{array}{l}\lambda_{\mathrm{bf}} \text { or } \lambda_{\mathrm{eff}}{ }^{\prime} \\
\left(\mathrm{mW} \cdot \mathrm{m}^{-1} \cdot \mathrm{K}^{-1}\right)\end{array}$ & $100 \cdot\left(\lambda_{\text {eff }}-\lambda_{\mathrm{bf}}\right) / \lambda_{\mathrm{bf}}$ \\
\hline \multirow[t]{4}{*}{$\mathrm{H}_{2} \mathrm{O}$} & 0 & 0 & 298.15 & 647.7 & 0.0 \\
\hline & & & 303.15 & 648.8 & 0.0 \\
\hline & & & 313.15 & 664.5 & 0.0 \\
\hline & & & 323.15 & 676.1 & 0.0 \\
\hline \multirow[t]{15}{*}{$\mathrm{TiO}_{2}+\mathrm{H}_{2} \mathrm{O}$} & 0.087 & 0.024 & 298.15 & 674.2 & 4.1 \\
\hline & & & 303.15 & 678.6 & 4.6 \\
\hline & & & 313.15 & 691.1 & 4.0 \\
\hline & & & 323.15 & 704.8 & 4.2 \\
\hline & & & $303.15^{\mathrm{a}}$ & 695.4 & 7.2 \\
\hline & 0.223 & 0.069 & 298.15 & 722.0 & 11.5 \\
\hline & & & 303.15 & 734.3 & 13.2 \\
\hline & & & 313.15 & 748.9 & 12.7 \\
\hline & & & 323.15 & 764.9 & 13.1 \\
\hline & & & $303.15^{\mathrm{a}}$ & 739.9 & 14.0 \\
\hline & 0.398 & 0.145 & 298.15 & 830.6 & 28.3 \\
\hline & & & 303.15 & 832.1 & 28.2 \\
\hline & & & 313.15 & 855.6 & 28.7 \\
\hline & & & 323.15 & 876.8 & 29.7 \\
\hline & & & $303.15^{\mathrm{a}}$ & 840.4 & 29.5 \\
\hline \multirow[t]{15}{*}{$\mathrm{SiO}_{2}+\mathrm{H}_{2} \mathrm{O}$} & 0.150 & 0.074 & 298.15 & 685.5 & 5.9 \\
\hline & & & 303.15 & 686.2 & 5.8 \\
\hline & & & 313.15 & 704.5 & 6.0 \\
\hline & & & 323.15 & 715.3 & 5.8 \\
\hline & & & $303.15^{\mathrm{a}}$ & 705.0 & 8.7 \\
\hline & 0.277 & 0.148 & 298.15 & 695.7 & 7.5 \\
\hline & & & 303.15 & 697.8 & 7.6 \\
\hline & & & 313.15 & 721.0 & 8.5 \\
\hline & & & 323.15 & 727.1 & 7.5 \\
\hline & & & $303.15^{\mathrm{a}}$ & 715.5 & 10.3 \\
\hline & 0.500 & 0.312 & 298.15 & 786.8 & 21.5 \\
\hline & & & 303.15 & 792.0 & 22.1 \\
\hline & & & 313.15 & 810.5 & 22.0 \\
\hline & & & 323.15 & 839.2 & 24.1 \\
\hline & & & $303.15^{\mathrm{a}}$ & 801.4 & 23.5 \\
\hline
\end{tabular}


Table 2 (continued)

\begin{tabular}{|c|c|c|c|c|c|}
\hline System & $w$ & $\varphi$ & $T / \mathrm{K}$ & $\begin{array}{l}\lambda_{\mathrm{bf}} \text { or } \lambda_{\mathrm{eff}} \mathrm{f} \\
\left(\mathrm{mW} \cdot \mathrm{m}^{-1} \cdot \mathrm{K}^{-1}\right)\end{array}$ & $100 \cdot\left(\lambda_{\text {eff }}-\lambda_{\mathrm{bf}}\right) / \lambda_{\mathrm{bf}}$ \\
\hline \multirow[t]{15}{*}{$\mathrm{PS}+\mathrm{H}_{2} \mathrm{O}$} & \multirow[t]{5}{*}{0.039} & \multirow[t]{5}{*}{0.037} & 298.15 & 593.0 & -8.4 \\
\hline & & & 303.15 & 582.2 & -10.3 \\
\hline & & & 313.15 & 602.4 & -9.3 \\
\hline & & & 323.15 & 608.3 & -10.0 \\
\hline & & & $303.15^{\mathrm{a}}$ & 608.1 & -6.3 \\
\hline & \multirow[t]{5}{*}{0.082} & \multirow[t]{5}{*}{0.078} & 298.15 & 567.8 & -12.3 \\
\hline & & & 303.15 & 575.0 & -11.4 \\
\hline & & & 313.15 & 588.7 & -11.4 \\
\hline & & & 323.15 & 584.0 & -13.6 \\
\hline & & & $303.15^{\mathrm{a}}$ & 588.5 & -9.3 \\
\hline & \multirow[t]{5}{*}{0.165} & \multirow[t]{5}{*}{0.158} & 298.15 & 483.7 & -25.3 \\
\hline & & & 303.15 & 493.3 & -24.0 \\
\hline & & & 313.15 & 514.1 & -22.6 \\
\hline & & & 323.15 & 518.2 & -23.4 \\
\hline & & & $303.15^{\mathrm{a}}$ & 504.2 & -22.3 \\
\hline
\end{tabular}

${ }^{\mathrm{a}}$ Repetition measurement 1 day later

in the experimental situation. The $\lambda_{\text {bf }}$ data obtained at $\Delta T=3 \mathrm{~K}$ are given in Table 2 and serve as a reference for the calculation of the enhancement factors for the effective thermal conductivity of the nanofluids.

For the three types of nanofluids, the measurement results for the effective thermal conductivity $\lambda_{\text {eff }}$ at ambient pressure are listed in Table 2 for the four studied temperatures of $(298.15,303.15,313.15$, and 323.15) $\mathrm{K}$ and the repetition measurement at $303.15 \mathrm{~K}$. From the measured $\lambda_{\text {eff }}$ and $\lambda_{\text {bf }}$ data with expanded uncertainties of $5 \%$, the percentage enhancement factor of the effective thermal conductivity relative to the thermal conductivity of the base fluid, $100 \cdot\left(\lambda_{\text {eff }}-\lambda_{b f}\right) / \lambda_{b f}$, can be calculated with an absolute expanded uncertainty of $7 \%(k=2)$ based on error propagation calculations. For the state points investigated for the nanofluids, the enhancement factors are listed in Table 2 and are illustrated in Fig. 6 as a function of the particle volume fraction. In the figure, different types of symbol fillings are used to differentiate between different nanofluid systems, while different symbol types represent different temperatures.

For all systems, a moderate and approximately linear change of the effective thermal conductivity and of the enhancement factor with increasing particle volume fraction is found. Within the temperature range investigated, no significant effect of temperature on the enhancement factor is given, as it has also been found in the experiments of Ehle et al. [31] with a modified version of a steady-state 


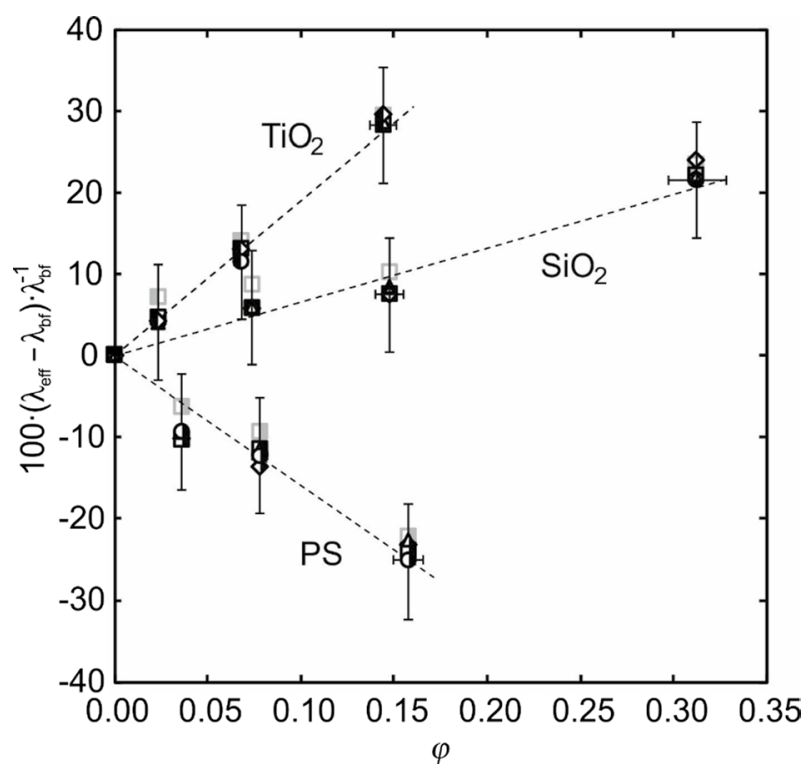

Fig. 6 Enhancement factors of the measured effective thermal conductivity of the three types of waterbased nanofluids relative to the thermal conductivity of water at different temperatures as a function of particle volume fraction: left-filled, $\mathrm{TiO}_{2}$; open, $\mathrm{SiO}_{2}$; right-filled, PS. Black circles, $298.15 \mathrm{~K}$, squares, 303.15 K; triangles, $313.15 \mathrm{~K}$; diamonds, $323.15 \mathrm{~K}$; gray squares, repetition at $303.15 \mathrm{~K}$. The uncertainties in the enhancement factor and in the particle volume fractions are only shown for the datasets measured at 298.15 K. Dashed lines displayed for each system serve as guides for the eye

concentric-cylinder instrument for the same type of initial nanofluid containing $\mathrm{SiO}_{2}$ particles. This finding indicates that the increasing Brownian motion of the particles with increasing temperature has a negligible effect on the effective thermal conductivity of nanofluids, which is in agreement with the HC model [18] and our model [21] discussed later on. The enhancement factor is positive for the $\mathrm{TiO}_{2}$ and $\mathrm{SiO}_{2}$ particles showing larger thermal conductivities $\left(\lambda_{\mathrm{p}} \approx 12 \mathrm{~W} \cdot \mathrm{m}^{-1} \cdot \mathrm{K}^{-1}\right.$ for $\mathrm{TiO}_{2}$, $\lambda_{\mathrm{p}} \approx 1.4 \mathrm{~W} \cdot \mathrm{m}^{-1} \cdot \mathrm{K}^{-1}$ for $\left.\mathrm{SiO}_{2}\right)$ than water $\left(\lambda_{\mathrm{bf}} \approx 0.6 \mathrm{~W} \cdot \mathrm{m}^{-1} \cdot \mathrm{K}^{-1}\right)$. Since $\mathrm{TiO}_{2}$ has a larger thermal conductivity than $\mathrm{SiO}_{2}$, the resulting enhancement factors for the $\mathrm{TiO}_{2}$-based nanofluids are also larger. In contrast, the enhancement factors for the PS-based systems are negative because of the lower thermal conductivity of PS $\left(\lambda_{\mathrm{p}} \approx 0.11 \mathrm{~W} \cdot \mathrm{m}^{-1} \cdot \mathrm{K}^{-1}\right)$ compared to water. Although in this case the term "enhancement" should be replaced by "reduction", we keep the nomenclature throughout the entire manuscript for the sake of convenience. The absolute reductions in the effective thermal conductivity of up to $-25 \%$ for the largest PS concentration are similar and somewhat larger than those obtained by the measurements of Rusconi et al. [35] for water-based nanofluids containing a different type of polymer particles $\left(\lambda_{\mathrm{p}} \approx 0.2 \mathrm{~W} \cdot \mathrm{m}^{-1} \cdot \mathrm{K}^{-1}\right)$ than studied in this work.

For all probed nanofluids at $303.15 \mathrm{~K}$, the effective thermal conductivities at the repetition measurements indicated by the gray markers in Fig. 6 showed only a slight increase by at most $3 \%$ relative to the first measurement at the previous day, which is within the experimental uncertainty. The repeatability of the measurements 
and, thus, the stability of the nanofluid samples was also confirmed by a long-time test over eight days for the most concentrated $\mathrm{TiO}_{2}$-based nanofluid with $\varphi=0.145$. For the entire period, the measured data recorded at each subsequent day matched within the measurement uncertainty and with the value from the first measurement set given in Table 2. In the following, only our measurement results at $298.15 \mathrm{~K}$ will be discussed because the effect of temperature on the effective thermal conductivity is negligible within the temperature range studied.

\subsubsection{Data Comparison}

A comparison of our measured effective thermal conductivities with experimental data from literature is only possible for the water-based nanofluids containing $\mathrm{TiO}_{2}$ or $\mathrm{SiO}_{2}$. For nanofluids with PS, no experimental data are available so far. In Fig. 7, the results for the enhancement factor obtained for the water-based nanofluids with nearly spherical $\mathrm{TiO}_{2}$ (left) and $\mathrm{SiO}_{2}$ (right) particles at comparable temperatures between (293.15 and 303.15) $\mathrm{K}$ are shown as a function of the particle volume fraction, where different markers detailed in the figure legend are used for the different literature sources. For the $\mathrm{TiO}_{2}$-based systems, only numerical values for $\lambda_{\text {eff }}$ or the resulting enhancement factors reported in literature were considered here, whereas a complete review of all available data is

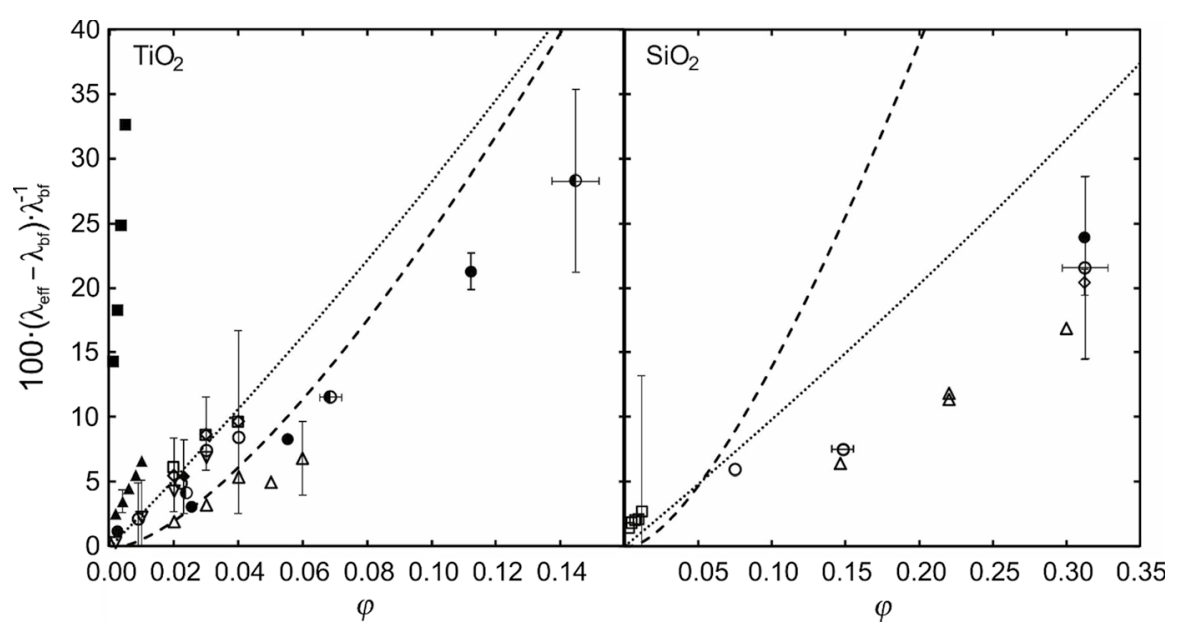

Fig. 7 Enhancement factors for the effective thermal conductivity of the water-based nanofluids containing spherical $\mathrm{TiO}_{2}$ (left) or $\mathrm{SiO}_{2}$ (right) particles at temperatures between (293.15 and 303.15) K. Experimental data for $\mathrm{TiO}_{2}$-based systems (left part): $\boldsymbol{D}, 66 \mathrm{~nm}, 298.15 \mathrm{~K}$, this work; $\bigcirc, 30$ to $50 \mathrm{~nm}$, $298.15 \mathrm{~K}$, Tertsinidou et al. [6]; $\Delta 5 \mathrm{~nm}, 298.15 \mathrm{~K}$, Tertsinidou et al. [6]; $\diamond, 5$ to $15 \mathrm{~nm}$ (with surfactant), $298.15 \mathrm{~K}$, Tertsinidou et al. [6]; $\square, 30$ to $50 \mathrm{~nm}$ (with surfactant), $298.15 \mathrm{~K}$, Tertsinidou et al. [6]; $\nabla, 21 \mathrm{~nm}, 296.15 \mathrm{~K}$, Turgut et al. [78]; •, 74 nm, $294.2 \mathrm{~K}$, Fedele et al. [62]; $\diamond, 20 \mathrm{~nm}, 293.15 \mathrm{~K}$, Haghighi et al. [77]; $\boldsymbol{\square}, 31 \mathrm{~nm}, 303 \mathrm{~K}$, Maheshwary et al. [80]; $\boldsymbol{\Delta}, 21 \mathrm{~nm}, 303.15 \mathrm{~K}$, Reddy and Rao [79]. Experimental data for $\mathrm{SiO}_{2}$-based systems (right part): $\bigcirc, 36 \mathrm{~nm}, 298.15 \mathrm{~K}$, this work; $\diamond, 20$ to $40 \mathrm{~nm}, 298.15$ to $303.15 \mathrm{~K}$, Buongiorno et al. [33]; $\square, 30 \mathrm{~nm}, 293.15 \mathrm{~K}$, Yang et al. [37]; $\Delta, 11$ to $30 \mathrm{~nm}, 293.15 \mathrm{~K}$, Turanov et al. [36]; •, $22 \mathrm{~nm}, \sim 299 \mathrm{~K}$, Ehle et al. [31]. Theoretical predictions: ..... , HC model [18]; - - - , our model [21]. Experimental uncertainties are exemplarily shown as error bars for the various datasets 
given by Tertsinidou et al. [5]. Considering the distinctly smaller database for the $\mathrm{SiO}_{2}$-based systems, available numerical values $[33,37]$ were supplemented with data extracted from figures $[31,36]$. The uncertainties of the enhancement factors exemplarily depicted in Fig. 7 for each dataset are based on error propagation calculations of the uncertainties of the $\lambda_{\text {eff }}$ and $\lambda_{b f}$ data. For comparison, the theoretical predictions based on the HC model [18] and our model [21] for nanofluids containing fully dispersed particles are shown as dotted and dashed lines. The origin of the thermophysical properties of the particles and the base fluid at atmospheric pressure needed for all subsequent calculations is summarized below.

For the thermal conductivity of the particles $\lambda_{\mathrm{p}}$ required in both models [18, $21]$ and assumed to be independent of size, values for $\mathrm{TiO}_{2}\left(\lambda_{\mathrm{p}}=12 \mathrm{~W} \cdot \mathrm{m}^{-1} \cdot \mathrm{K}^{-1}\right.$ at $298.15 \mathrm{~K})$ [5], for $\mathrm{SiO}_{2}\left(\lambda_{\mathrm{p}}=1.4-1.8 \mathrm{~W} \cdot \mathrm{m}^{-1} \cdot \mathrm{K}^{-1}\right.$ from $273 \mathrm{~K}$ to $\left.463 \mathrm{~K}\right)$ [43], and for PS ( $\lambda_{\mathrm{p}}=0.105-0.128 \mathrm{~W} \cdot \mathrm{m}^{-1} \cdot \mathrm{K}^{-1}$ from $273 \mathrm{~K}$ to $\left.373 \mathrm{~K}\right)$ [76] were taken from literature. Here, the corresponding $\lambda_{\mathrm{p}}$ data for $\mathrm{SiO}_{2}$ and PS were correlated linearly as a function of temperature within the specified range to calculate the values at $298.15 \mathrm{~K}$. For the particle density $\rho_{\mathrm{p}}$ in our model [21], the same data at $298.15 \mathrm{~K}$ as detailed in Sect. 2.1 were used. For the calculation of the Nußelt number $N u$ in the model [21], the values for the thermal conductivity $\lambda_{\mathrm{bf}}$, density $\rho_{\mathrm{bf}}$, kinematic viscosity $\nu_{\mathrm{bf}}$, and thermal diffusivity $a_{\mathrm{bf}}$ of the base fluid water specified by the Refprop database [42] were employed. Here, the required information about the diameter of the considered spherical particles was approximated with the mean values $d_{50,3}$ obtained by the sedimentation analysis and used for all particle volume fractions studied. This is justified because the mean diameters obtained from the DLS measurements close to infinite dilution and from the sedimentation analysis for the most concentrated nanofluids agree within combined uncertainties.

The left part of Fig. 7 shows for the $\mathrm{TiO}_{2}$-based systems with a $\lambda_{\mathrm{p}} / \lambda_{\mathrm{bf}}$ ratio of about 20 that most of the experimental data in literature $[6,62,77,78]$ associated with particle volume fractions up to 0.06 provide moderate enhancement factors which scatter around the HC model [18] and our model [21]. Only the data obtained by Reddy and Rao [79] with the steady-state concentric-cylinder method with a stated uncertainty of $0.6 \%$ and especially those obtained by Maheshway et al. [80] with a hot-wire method of unknown uncertainty show clearly larger enhancement factors. Our measurement results follow the data of Fedele et al. [62] based on the hot-disk technique and extend the data range up to larger particle volume fractions. Here, for $\varphi>0.06$, the available experimental data are in better agreement with our model [21] than with the HC model [18]. The lower measured enhancements factors than predicted by the $\mathrm{HC}$ model indicates that an additional thermal resistance related to the interface is present, which seems to be reflected appropriately in our model [21] by additionally considering the convective heat transfer between the fluid and the nanoparticles.

For the $\mathrm{SiO}_{2}$-based systems featuring a $\lambda_{\mathrm{p}} / \lambda_{\mathrm{bf}}$ ratio of about 2.5 , the four different experimental datasets $[31,33,36,37]$ including our data shown in the right part of Fig. 7 agree well with each other over the entire concentration range up to $\varphi$ values of about 0.3. For the latter concentration corresponding to the same type of sample 


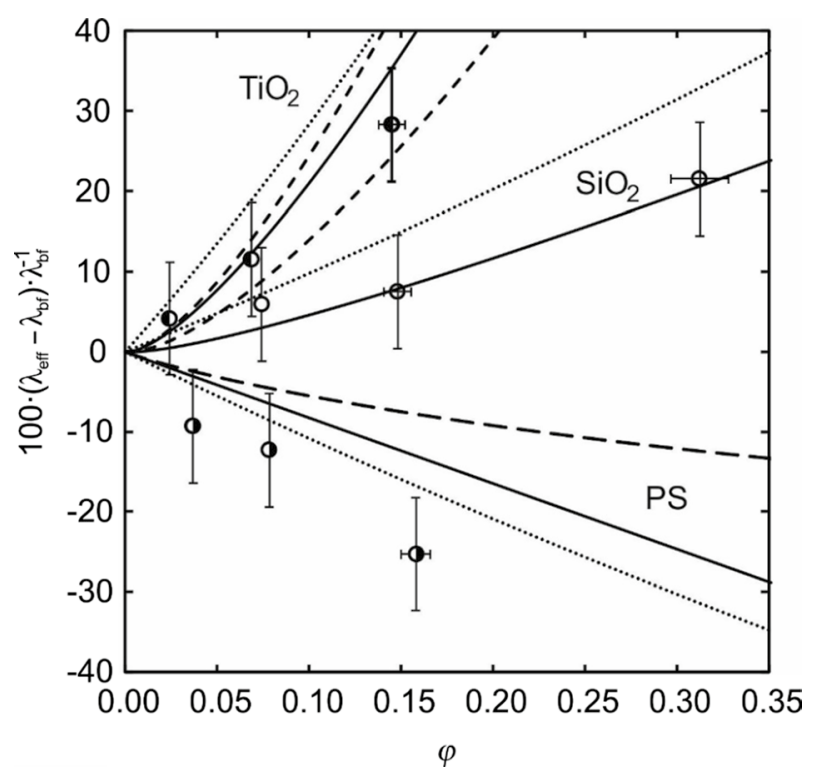

Fig. 8 Comparison of enhancement factors of the measured effective thermal conductivities of the waterbased nanofluids containing spherical $\mathrm{TiO}_{2}, \mathrm{SiO}_{2}$, or PS particles at $298.15 \mathrm{~K}$ with the predictions from different modeling approaches as a function of the particle volume fraction. Experimental data from this work: $\mathbf{D}, \mathrm{TiO}_{2} ; \mathrm{O}, \mathrm{SiO}_{2} ; \mathbf{O}, \mathrm{PS}$; Theoretical predictions: ...... HC model [18]; — - - , our model [21]; — , improved model according to Eq. 3 from this work

investigated in a benchmark study of Buongiorno et al. [33] by different methods including a parallel-plate method, whose working procedure has not been described, and by Ehle et al. [31] with a concentric-cylinder instrument requiring a calibration, matching enhancement factors with relative absolute deviations of $1.1 \%$ and $2.5 \%$ from our measurement result are found. In addition, the measurement results at $\varphi$ values larger than 0.1 give a weaker concentration-dependent trend than the $\mathrm{HC}$ model [18], which is similar to the situation found for the $\mathrm{TiO}_{2}$-based systems, and especially than our model [21]. Both models diverge for particle volume fractions above 0.05 . It seems that our existing model can represent the behavior of the enhancement factor for nanofluids with relatively large $\lambda_{\mathrm{p}} / \lambda_{\mathrm{bf}}$ ratios well, as it is given for the present $\mathrm{TiO}_{2}$-based nanofluids and for the nanofluids studied in our previous work [21]. However, the model overestimates the enhancement factors for decreasing $\lambda_{\mathrm{p}} / \lambda_{\mathrm{bf}}$ ratios, i.e., for particles with relatively small thermal conductivities such as $\mathrm{SiO}_{2}$. The same trend is also found for the nanofluids containing PS particles with a $\lambda_{\mathrm{p}} / \lambda_{\mathrm{bf}}$ ratio of about 0.2 , which will be discussed later in connection with Fig. 8.

\subsubsection{Improvement of Prediction Method}

The existing discrepancy between our experimental and theoretical data for systems with relatively low $\lambda_{\mathrm{p}} / \lambda_{\mathrm{bf}}$ ratios motivated an improvement of our prediction method for the effective thermal conductivity of nanofluids. Before the description of the 
improvements, the main concept of the model is summarized in the following. More details can be found in Ref. [21].

In our model proposed in 2016, the heat transfer problem in nanofluids containing fully dispersed particles is treated by the analysis of the corresponding thermal resistances present in such systems. For this, a single nanoparticle with diameter or volume-equivalent diameter for spherical or non-spherical particles, $d_{\mathrm{p}}$, is located in a cube which is filled with the liquid and has an edge length of $L$. By this, the particle volume fraction $\varphi=(\pi / 6) \cdot\left(d_{\mathrm{p}} / L\right)^{3}$ is defined. To calculate the total thermal resistance of the nanofluid, $R_{\text {eff }}$, connected with its effective thermal conductivity $\lambda_{\text {eff }}$, the thermal resistance of the base fluid, $R_{\mathrm{bf}}$, is considered to be parallel to the thermal resistance related to the nanoparticle, $R_{\mathrm{p}}$. The latter is modeled by the sum of the thermal resistance caused by the thermal conduction in the particles, $R_{\mathrm{p}, \text { cond }}$, and by the convective heat transfer through the boundary layer at the surface of the nanoparticles, $R_{\mathrm{p}, \mathrm{conv}}$. To account for the volumes associated with the resistances of the two phases, the inverse values of the thermal resistances $R_{\mathrm{bf}}$ and $R_{\mathrm{p}}$ are weighted by the corresponding volume fractions $(1-\varphi)$ and $\varphi$, resulting in

$$
\frac{1}{R_{\mathrm{eff}}}=\frac{1-\varphi}{R_{\mathrm{bf}}}+\frac{\varphi}{R_{\mathrm{p}, \mathrm{conv}}+R_{\mathrm{p}, \mathrm{cond}}}
$$

By inserting the three individual resistance contributions into Eq. 2, an analytical model for the effective thermal conductivity could be derived as a function of the volume fraction, diameter, and shape of the nanoparticles, the thermal conductivities of the particles and of the base fluid as well as the temperature [21]. While the particle diameter and temperature have no significant effect on the enhancement factor, the latter increases with increasing $\lambda_{\mathrm{p}} / \lambda_{\mathrm{bf}}$ ratios and changes steadily with increasing $\varphi$ values.

According to the observations made in connection with Fig. 7, the reason behind the failure of our model for nanofluids containing particles of relatively low thermal conductivities seems to be related to the resistance due to the thermal conduction through the particles, $R_{\mathrm{p}, \text { cond }}$. With decreasing $\lambda_{\mathrm{p}}$ values, $R_{\mathrm{p}, \text { cond }}$ increases and can become larger than $R_{\mathrm{p}, \text { conv }}$, i.e., $R_{\mathrm{p} \text {,cond }}$ is the limiting factor for the thermal resistance related to the nanoparticle, $R_{\mathrm{p}}$. Thus, our current model apparently underestimates the value for $R_{\mathrm{p}, \text { cond }}$ which is effectively modeled by the thermal resistance of an isothermal nanoparticle, $R_{\mathrm{p}, \text { cond }}=1 /\left(2 \pi \cdot \lambda_{\mathrm{p}} \cdot d_{\mathrm{p}}\right)$. In an analogous way as it has been performed for $R_{\mathrm{bf}}$ in our former approach [21], it is more appropriate to model $R_{\mathrm{p} \text {,cond }}$ by $R_{\mathrm{p}, \text { cond }}=L /\left(\lambda_{\mathrm{p}} \cdot L^{2}\right)=1 /\left(\lambda_{\mathrm{p}} \cdot L\right)$, taking into consideration the length $L$ and the cross-section $L^{2}$ of the cube which consists now solely of the particle. Since $R_{\mathrm{p} \text {,cond }}$ is inversely weighted by the particle volume fraction $\varphi$ in Eq. 2, the contribution of the thermal conduction through the particle to the overall effective conduction in the nanoparticle-fluid system is already considered reasonably. It is found that starting from $\varphi \approx 0.002$, the reformulated $R_{\mathrm{p} \text {,cond }}$ value is always larger than the former one, which increases with increasing $\varphi$.

On the basis of the new definition for $R_{\mathrm{p}, \text { cond }}$ and the same definitions for $R_{\mathrm{eff}}$, $R_{\mathrm{bf}}$, and $R_{\mathrm{p}, \text { conv }}$ as given in our former work [21], the revisited model for the 
dimensionless effective thermal conductivity $\lambda_{\text {eff }} / \lambda_{\text {bf }}$ of nanofluids containing fully dispersed particles has the simple form

$$
\frac{\lambda_{\mathrm{eff}}}{\lambda_{\mathrm{bf}}}=(1-\varphi)+\varphi\left[\frac{\lambda_{\mathrm{bf}}}{\lambda_{\mathrm{p}}}+\left(\frac{\pi}{6 \varphi}\right)^{1 / 3} \cdot \frac{\psi}{\pi \cdot \mathrm{Nu}}\right]^{-1} .
$$

In Eq. 3, $\psi$ is the sphericity of the particles, which is 1 for spherical particles. For particles of same volume, decreasing sphericity of the particles $(\psi<1)$ causes an increasing surface area and, thus, a decreasing value for $R_{\mathrm{p}, \text { conv }}$, which increases the enhancement factor according to Eq. 3. Nu is the Nußelt number which can be obtained from a correlation of the Péclet number $(P e)$ and, thus, from the Prandtl $(P r)$ and Reynolds $(R e)$ numbers [21]. For macroscopically static nanofluids, $P e<1$ and $R e \ll 1$, which results in $N u$ numbers close to 2 . In the improved model, the same equations were applied to calculate the $N u$ number, as it has been performed in our previous work [21]. Furthermore, the experimental $d_{50,3}$ values were used as input to calculate the $R e$ and, thus, $N u$ numbers.

In Fig. 8, our measured enhancement factors for the effective thermal conductivity of the nanofluids studied at a temperature of $298.15 \mathrm{~K}$ are compared with the theoretical predictions for well-dispersed systems based on the HC model (dotted line) [18], our model (dashed line) [21], and its revisited improved version given by Eq. 3 (solid line) for varying volume fractions of the spherical particles. For all nanofluid systems studied, the improved model provides a better representation of our concentration-dependent experimental data in comparison with the original model. This holds in particular for the water-based nanofluids containing $\mathrm{SiO}_{2}$ or PS particles with small $\lambda_{\mathrm{p}} / \lambda_{\mathrm{bf}}$ ratios of 2.5 or 0.2 , where the reformulated expression for the resistance of the nanoparticles due to conduction reduces the enhancement factor more strongly. For all cases, the improved model is also in good agreement with the HC model [18]. In this model, it is assumed that only thermal conduction is present, i.e., no thermal resistance exists at the interface between the liquid and the solid phase. The latter is taken into consideration in our model via the convective heat transfer resistance between the fluid and the diffusing particles, $R_{\mathrm{p}, \mathrm{conv}}$. As a result, smaller enhancement factors are predicted by our model than by the HC model [18] for the $\mathrm{TiO}_{2}$ - and $\mathrm{SiO}_{2}$-based systems where $R_{\mathrm{p}, \text { conv }}$ is distinctly larger than or comparable to $R_{\mathrm{p} \text {,cond }}$.

When $R_{\mathrm{p} \text {,cond }}$ is much larger than $R_{\mathrm{p} \text {,conv }}$, as it is the case for the PS-based systems, the improved model provides slightly larger enhancement factors than the HC model [18]. In this case where the dispersed nanoparticles act as thermal insulators, the heat flux is mainly transferred through the continuous liquid phase, resulting in predictions close to those for a simple parallel conduction model [50]. The residual discrepancy between the predictions from our improved model and the measured data may arise from a weak sedimentation of the PS particles within the guarded parallel-plate instrument. This behavior corresponds to some extent to a serial conduction model which gives distinctly lower values for $\lambda_{\text {eff }}$ and, thus, for the enhancement factors than the parallel conduction model [50]. 
It should be noted that the predictions based on our improved model are also in good agreement with the approach of Nan et al. [20] who included a term accounting for the interfacial thermal contact resistance $R_{\mathrm{b}}$ between the particles and the fluid within the $\mathrm{HC}$ model. For example, using the value for $R_{\mathrm{b}}$ derived by Ehle et al. [31] from their measurements for the most concentrated $\mathrm{SiO}_{2}$-based nanofluid in combination with the values for $d_{50,3}$ and $k_{\mathrm{p}}$ of the assumed spherical nanoparticles, the model of Nan et al. [20] showing smaller enhancement factors than the HC model agrees with our improved model and our experimental data within $8 \%$ over the entire concentration range studied. The same trends can be found for the $\mathrm{TiO}_{2}$-based nanofluids using a typical value for $R_{\mathrm{b}}$ for nanofluid systems [33] in the model of Nan et al. [20]. All these findings imply that the convective heat transfer resistance between the fluid and the particles included in our model and related to the term associated with the $N u$ number in Eq. 3 seems to reasonably account for an interfacial thermal contact resistance.

\section{Conclusions}

In the present work, the effective thermal conductivity of nanofluids consisting of nearly spherical and monodisperse titanium dioxide $\left(\mathrm{TiO}_{2}\right)$, silicon dioxide $\left(\mathrm{SiO}_{2}\right)$, or polystyrene (PS) nanoparticles and water as liquid continuous phase was investigated. It could be demonstrated for the first time that a steady-state guarded parallel-plate instrument can be applied reliably for the absolute determination of the effective thermal conductivity of dispersions over a broad composition range up to particle volume fractions of 0.31 . For the same systems, DLS experiments analyzing the translational diffusion coefficients in macroscopic thermodynamic equilibrium could reveal the stability of the nanofluids by the agreement of the derived particle sizes and their distributions with the results from scanning electron microscopy and differential centrifugal sedimentation measurements. The experimental data for the effective thermal conductivity with an expanded uncertainty of $5 \%$ show no significant dependence on temperature between (298.15 and 323.15) $\mathrm{K}$ and a moderate change as a function of the particle volume fraction. For the systems with $\mathrm{TiO}_{2}$ or $\mathrm{SiO}_{2}$ particles showing larger thermal conductivities than water, the corresponding enhancement factors of the effective thermal conductivity are positive and agree well with the available literature. For the systems containing PS particles of lower thermal conductivity compared to that of water, the measured negative enhancement factors are the first data reported so far. In connection with the nanofluids containing the relatively weakly conductive $\mathrm{SiO}_{2}$ and PS particles, the overestimations of the enhancement factors by our model proposed in 2016 [21] could be related to the used expression for the thermal resistance associated with the thermal conduction through the particles. Based on a revisited formulation for the latter resistance, our model for the effective thermal conductivity of nanofluids with fully dispersed particles could be improved and enables now reliable predictions in case of dispersed particles with broadly varying thermal conductivities. 
Acknowledgments Open Access funding provided by Projekt DEAL. This work was funded by the Deutsche Forschungsgemeinschaft (DFG, German Research Foundation)_Project-ID 416229255-SFB 1411. Furthermore, financial support of DFG by funding the Erlangen Graduate School in Advanced Optical Technologies (SAOT) within the German Excellence Initiative is acknowledged.

Open Access This article is licensed under a Creative Commons Attribution 4.0 International License, which permits use, sharing, adaptation, distribution and reproduction in any medium or format, as long as you give appropriate credit to the original author(s) and the source, provide a link to the Creative Commons licence, and indicate if changes were made. The images or other third party material in this article are included in the article's Creative Commons licence, unless indicated otherwise in a credit line to the material. If material is not included in the article's Creative Commons licence and your intended use is not permitted by statutory regulation or exceeds the permitted use, you will need to obtain permission directly from the copyright holder. To view a copy of this licence, visit http://creativecommons.org/licen ses/by/4.0/.

\section{References}

1. M.H. Buschmann, R. Azizian, T. Kempe, J.E. Juliá, R. Martínez-Cuenca, B. Sundén, Z. Wu, A. Seppälä, T. Ala-Nissila, Int. J. Thermal Sci. 129, 504-531 (2018)

2. M. Schwan, L.G. Kramer, T. Sottmann, R. Strey, Phys. Chem. Chem. Phys. 12, 6247-6252 (2010)

3. M.M. MacDevette, T.G. Myers, Int. J. Heat Mass Transf. 92, 550-557 (2016)

4. S.U.S. Choi, Z.G. Zhang, W. Yu, F.E. Lockwood, E.A. Grulke, Appl. Phys. Lett. 79, 2252-2254 (2001)

5. G. Tertsinidou, M.J. Assael, W.A. Wakeham, Int. J. Thermophys. 36, 1367-1395 (2015)

6. G.J. Tertsinidou, C.M. Tsolakidou, M. Pantzali, M.J. Assael, L. Colla, L. Fedele, S. Bobbo, W.A. Wakeham, J. Chem. Eng. Data 62, 491-507 (2016)

7. P. Keblinski, R. Prasher, J. Eapen, J. Nanopart. Res. 10, 1089-1097 (2008)

8. S.P. Jang, S.U.S. Choi, Appl. Phys. Lett. 84, 4316-4318 (2004)

9. J. Koo, C. Kleinstreuer, J. Nanopart. Res. 6, 577-588 (2005)

10. R. Prasher, P. Bhattacharya, P.E. Phelan, J. Heat Transf. 128, 588-595 (2006)

11. J.W. Gao, R.T. Zheng, H. Ohtani, D.S. Zhu, G. Chen, Nano Lett. 9, 4128-4132 (2009)

12. E.V. Timofeeva, A.N. Gavrilov, J.M. McCloskey, Y.V. Tolmachev, S. Sprunt, L.M. Lopatina, J.V. Selinger, Phys. Rev. E 76, 061203 (2007)

13. W. Yu, S.U.S. Choi, J. Nanopart. Res. 6, 355-361 (2004)

14. H.U. Kang, S.H. Kim, J.M. Oh, Exp. Heat Transf. 19, 181-191 (2006)

15. H.E. Patel, T. Sundararajan, S.K. Das, J. Nanopart. Res. 12, 1015-1031 (2009)

16. C.H. Li, G.P. Peterson, J. Appl. Phys. 99, 084314 (2006)

17. M. Corcione, Energy Convers. Manag. 52, 789-793 (2011)

18. R.L. Hamilton, O.K. Crosser, Ind. Eng. Chem. Fundam. 1, 187-191 (1962)

19. Y. Xuan, Q. Li, W. Hu, Thermodynamics 49, 1038-1043 (2003)

20. C.-W. Nan, R. Birringer, D.R. Clarke, H. Gleiter, J. Appl. Phys. 81, 6692-6699 (1997)

21. K.N. Shukla, T.M. Koller, M.H. Rausch, A.P. Fröba, Int. J. Heat Mass Transf. 99, 532-540 (2016)

22. S.M.S. Murshed, K.C. Leong, C. Yang, Int. J. Therm. Sci. 47, 560-568 (2008)

23. M.P. Beck, Y. Yuan, P. Warrier, A.S. Teja, J. Nanopart. Res. 12, 1469-1477 (2009)

24. G.A. Longo, C. Zilio, Int. J. Thermophys. 34, 1288-1307 (2013)

25. G.A. Longo, C. Zilio, Exp. Therm. Fluid Sci. 35, 1313-1324 (2011)

26. S.K. Das, N. Putra, P. Thiesen, W. Roetzel, J. Heat Transf. 125, 567-574 (2003)

27. R. Karthik, R. Nagarajan, B. Raja, P. Damodharan, Exp. Therm. Fluid Sci. 40, 1-9 (2012)

28. D.-W. Oh, A. Jain, J.K. Eaton, K.E. Goodson, J.S. Lee, Int. J. Heat Fluid Flow 29, 1456-1461 (2008)

29. H.C.B. Costa, D.O. Silva, L.G.M. Vieira, Heat Mass Transf. 55, 669-683 (2018)

30. B. Barbés, R. Páramo, F. Sobrón, E. Blanco, C. Casanova, J. Therm. Anal. Calorim. 104, 805-812 (2010)

31. A. Ehle, S. Feja, M.H. Buschmann, J. Thermophys. Heat Transf. 25, 378-385 (2011)

32. X. Wang, X. Xu, S.U.S. Choi, J. Thermophys. Heat Transf. 13, 474-480 (1999) 
33. J. Buongiorno, D.C. Venerus, N. Prabhat, T. McKrell, J. Townsend, R. Christianson, Y.V. Tolmachev, P. Keblinski, L.-W. Hu, J.L. Alvarado, I. Cheol Bang, S.W. Bishnoi, M. Bonetti, F. Botz, A. Cecere, Y. Chang, G. Chen, H. Chen, S.J. Chung, M.K. Chyu, S.K. Das, R.D. Paola, Y. Ding, F. Dubois, G. Dzido, J. Eapen, W. Escher, D. Funfschilling, Q. Galand, J. Gao, P.E. Gharagozloo, K.E. Goodson, J.G. Gutierrez, H. Hong, M. Horton, K.S. Hwang, C.S. Iorio, S.P. Jang, A.B. Jarzebski, Y. Jiang, L. Jin, S. Kabelac, A. Kamath, M.A. Kedzierski, L.G. Kieng, C. Kim, J.-H. Kim, S. Kim, S.H. Lee, K.C. Leong, I. Manna, B. Michel, R. Ni, H.E. Patel, J. Philip, D. Poulikakos, C. Reynaud, R. Savino, P.K. Singh, P. Song, T. Sundararajan, E. Timofeeva, T. Tritcak, A.N. Turanov, S.V. Vaerenbergh, D. Wen, S. Witharana, C. Yang, W.-H. Yeh, X.-Z. Zhao, S.-Q. Zhou, J. Appl. Phys. 106, $094312(2009)$

34. Y. Hwang, J.K. Lee, C.H. Lee, Y.M. Jung, S.I. Cheong, C.G. Lee, B.C. Ku, S.P. Jang, Thermochim. Acta 455, 70-74 (2007)

35. R. Rusconi, E. Rodari, R. Piazza, Appl. Phys. Lett. 89, 261916 (2006)

36. A.N. Turanov, Y.V. Tolmachev, Heat Mass Transf. 45, 1583-1588 (2009)

37. X.-F. Yang, Z.-H. Liu, Nanoscale Res. Lett. 6, 494 (2011)

38. AERODISP ${ }^{\circledR}$ W-740 X Datasheet. https://products-re.evonik.com/www2/uploads/productfinder/ AERODISP-W-740-X-DE.pdf. Accessed 14 Dec 2019

39. LUDOX ${ }^{\circledR}$ TM-50 colloidal silica Datasheet. https://www.sigmaaldrich.com/Graphics/COfAInfo/ SigmaSAPQM/SPEC/42/420778/420778-BULK ALDRICH_.pdf. Accessed 14 Dec 2019

40. LUDOX $^{\circledR}$ TM-50 colloidal silica Datasheet. https://grace.com/coatings-and-inks/en-us/Documents/ LUDOX\%20Coatings\%20TI.pdf (accessed December 14, 2019)

41. J. Zhang, Z. Chen, Z. Wang, W. Zhang, N. Ming, Mat. Lett. 57, 4466-4470 (2003)

42. E.W. Lemmon, M.L. Huber, M.O. McLinden, NIST Reference Fluid Thermodynamic and Transport Properties Database (REFPROP); version 9.1; NIST Standard Reference Database 23; National Institute of Standards and Technology: Gaithersburg, MD, 2013

43. D.R. Lide, CRC Handbook of Chemistry and Physics, 85th edn. (Taylor \& Francis, New York, 2004)

44. D.W. van Krevelen, K. te Nijenhuis, Properties of polymers: Their correlation with chemical structure; their numerical estimation and prediction from additive group contributions, 4th edn. (Elsevier, Amsterdam, 2009)

45. A.P. Fröba, M.H. Rausch, K. Krzeminski, D. Assenbaum, P. Wasserscheid, A. Leipertz, Int. J. Thermophys. 31, 2059-2077 (2010)

46. T.M. Koller, S.R. Schmid, S.J. Sachnov, M.H. Rausch, P. Wasserscheid, A.P. Fröba, Int. J. Thermophys. 35, 195-217 (2014)

47. M.H. Rausch, K. Krzeminski, A. Leipertz, A.P. Fröba, Int. J. Heat Mass Transf. 58, 610-618 (2013)

48. Y.M. Naziev, M.M. Bashirov, I.M. Abdulagatov, Fluid Phase Equilib. 226, 221-235 (2004)

49. H.A. Pakravan, M. Yaghoubi, Int. J. Therm. Sci. 50, 394-402 (2011)

50. J. Eapen, R. Rusconi, R. Piazza, S. Yip, J. Heat Transf. 132, 102402 (2010)

51. R. Savino, D. Paterna, Phys. Fluids 20, 017101 (2008)

52. A.P. Fröba, A. Leipertz, Diff. Fund. 63, 1-25 (2005)

53. M. Piszko, W. Wu, S. Will, M.H. Rausch, C. Giraudet, A.P. Fröba, Fuel 242, 562-572 (2019)

54. W. Wu, T. Klein, M. Kerscher, M.H. Rausch, T.M. Koller, C. Giraudet, A.P. Fröba, J. Phys. Chem. B 123, 8777-8790 (2019)

55. T. Klein, W. Wu, M.H. Rausch, C. Giraudet, T.M. Koller, A.P. Fröba, J. Phys. Chem. B 122, $7122-$ 7133 (2018)

56. M. Piszko, K. Batz, M.H. Rausch, C. Giraudet, A.P. Fröba, J. Chem. Eng. Data (2019). https://doi. org/10.1021/acs.jced.9b00495

57. S. Bi, T.M. Koller, M.H. Rausch, P. Wasserscheid, A.P. Fröba, Ind. Eng. Chem. Res. 54, 3071-3081 (2015)

58. M.S.G. Knoll, C. Giraudet, C.J. Hahn, M.H. Rausch, A.P. Fröba, J. Colloid Interf. Sci. 544, 144154 (2019)

59. F.E. Berger Bioucas, C. Damm, W. Peukert, M.H. Rausch, T.M. Koller, C. Giraudet, A.P. Fröba, J. Phys. Chem. B 123, 9491-9502 (2019)

60. B.J. Berne, R. Pecora, Dynamic light scattering: with applications to chemistry, biology, and physics (Dover Publications, Courier Corporation, Chelmsford, 2000)

61. A. Einstein, Z. Elektrochem, Angew. Phys. Chem. 14, 235-239 (1908)

62. L. Fedele, L. Colla, S. Bobbo, Int. J. Refrig 35, 1359-1366 (2012) 
63. M.M. Kops-Werkhoven, H.M. Fijnaut, J. Chem. Phys. 74, 1618-1625 (1981)

64. G.K. Batchelor, J. Fluid Mech. 74, 1-29 (1976)

65. C.W.J. Beenakker, P. Mazur, Physica 126A, 349-370 (1984)

66. T.P. Dale, J.H. Gladstone, Philos. Trans. Royal Soc. 148, 887-894 (1858)

67. T.P. Dale, J.H. Gladstone, Philos. Trans. Royal Soc. 153, 317-343 (1863)

68. M.H. Rausch, A. Heller, J. Herbst, T.M. Koller, M. Bahlmann, P.S. Schulz, P. Wasserscheid, A.P. Fröba, J. Phys. Chem. B 118, 4636-4646 (2014)

69. A.H. Harvey, J.S. Gallagher, J.M.H.L. Sengers, J. Phys. Chem. Ref. Data 27, 761-774 (1998)

70. J. Rumble, CRC handbook of chemistry and physics, 99th edn. (Taylor \& Francis, New York, 2018)

71. N. Sultanova, S. Kasarova, I. Nikolov, Acta Phys. Pol., A 116, 585-587 (2009)

72. M.L. Huber, R.A. Perkins, A. Laesecke, D.G. Friend, J.V. Sengers, M.J. Assael, I.N. Metaxa, E. Vogel, R. Mareš, K. Miyagawa, J. Phys. Chem. Ref. Data 38, 101-125 (2009)

73. A.L. Kholodenko, J.F. Douglas, Phys. Rev. E 51, 1081-1090 (1995)

74. B. Cichocki, B.U. Felderhof, Phys. Rev. A 46, 7723-7732 (1992)

75. M.L. Huber, R.A. Perkins, D.G. Friend, J.V. Sengers, M.J. Assael, I.N. Metaxa, K. Miyagawa, R. Hellmann, E. Vogel, J. Phys. Chem. Ref. Data 41, 033102 (2012)

76. J.E. Mark, Polymer data handbook, 1st edn. (Oxford University Press, Oxford, 1999)

77. E.B. Haghighi, M. Saleemi, N. Nikkam, R. Khodabandeh, M.S. Toprak, M. Muhammed, B. Palm, Int. Comm. Heat Mass Transf. 52, 1-7 (2014)

78. A. Turgut, I. Tavman, M. Chirtoc, H.P. Schuchmann, C. Sauter, S. Tavman, Int. J. Thermophys. 30, 1213-1226 (2009)

79. M.C.S. Reddy, V.V. Rao, Int. Comm. Heat Mass Transf. 46, 31-36 (2013)

80. P.B. Maheshwary, C.C. Handa, K.R. Nemade, Appl. Therm. Eng. 119, 79-88 (2017)

Publisher's Note Springer Nature remains neutral with regard to jurisdictional claims in published maps and institutional affiliations.

\section{Affiliations}

\section{Francisco E. Berger Bioucas ${ }^{1}$. Michael H. Rausch ${ }^{1}$. Jochen Schmidt ${ }^{2}$. Andreas Bück ${ }^{2}$. Thomas M. Koller ${ }^{1}$ (1) . Andreas P. Fröba ${ }^{1}$}

1 Institute of Advanced Optical Technologies-Thermophysical Properties (AOT-TP), Department of Chemical and Biological Engineering (CBI) and Erlangen Graduate School in Advanced Optical Technologies (SAOT), Friedrich-Alexander-University Erlangen-Nürnberg (FAU), Paul-Gordan-Straße 8, 91052 Erlangen, Germany

2 Institute of Particle Technology (LFG), Department of Chemical and Biological Engineering (CBI), Friedrich-Alexander-University Erlangen-Nürnberg (FAU), Cauerstraße 4, 91058 Erlangen, Germany 